\title{
The effectiveness of transvenous leads extractions implanted more than 10 years before
}

Aleksander Maciąg ${ }^{1}$, Paweł Syska ${ }^{1}$, Andrzej Przybylski ${ }^{2}$, Krzysztof Kuśmierski ${ }^{3}$, Maciej Sterliński ${ }^{1}$, Artur Oręziak ${ }^{2}$, Mariusz Pytkowski ${ }^{1}$, Jacek Różański ${ }^{3}$, Hanna Szwed ${ }^{1}$

${ }^{1} 2^{\text {nd }}$ Coronary Disease Department, Institute of Cardiology, Warsaw, Poland

${ }^{2}$ Arrhythmia Department, Institute of Cardiology, Warsaw, Poland

${ }^{3}$ Cardiosurgery and Transplantology Department, Institute of Cardiology, Warsaw, Poland

\begin{abstract}
Background: The increasing number of patients with cardiac implantable electronic devices (CIEDs) causes a rise in the absolute percentage of individuals qualifying for a transvenous lead extraction (TLE) due to infectious, vascular or lead failure related indications. As the survival time prolongs, TLE procedures more and more often concern the electrodes of long-term functioning. Authors provide a retrospective analysis of the effectiveness and safety of TLE performed on leads implanted at least 10 years before the extraction.

Methods: Between 2008 and 2012 we performed TLE of 364 electrodes in 217 patients. Out of these, 66 (18.1\%) leads in 43 (19.8\%) patients had been implanted for at least 10 years. The mean dwelling time for electrodes was 161 months (120 to 330). In $62 \%$ of cases CIED-related infection was an indication for TLE. The following extracting techniques were used: manual direct traction, device traction, mechanical telescopic sheaths, autorotational cutting sheaths and femoral approach.
\end{abstract}

Results: Fifty-eight pacemakers and 8 defibrillating leads were extracted. Sixty-three (95\%) completely, in the remaining 3 cases the clinical success was achieved with the small portion of the lead left into the vascular space. No major procedure complications were observed; minor complications were found in $3(6 \%)$ patients.

Conclusions: TLE with the use of various endovascular techniques is an effective and safe method for treating infectious, vascular and mechanical complications of long-lasting CIEDs therapy. (Cardiol J 2014; 21, 4: 419-424)

Key words: lead extraction, pacing and defibrillation leads

\section{Introduction}

The increasing number of patients with cardiac implantable electronic devices (CIEDs) is a challenge in terms of organizing a professional health care system for this group. With the prolonging survival period and an increasing number of multi-lead system (e.g. cardiac resynchronization therapy), the absolute percentage of complications rises. Some of these complications constitute an indication for transvenous lead extraction (TLE). The CIED-related infections require complete device removal. The lead-only removal should be considered in patients with central venous occlusion, in patients with damaged leads with an indication for lead exchange, in patients with lead excess

Address for correspondence: Aleksander Maciag, $\mathrm{MD}, \mathrm{PhD}, 2^{\text {nd }}$ Coronary Disease Department, Institute of Cardiology, ul. Spartańska 1, 02-637 Warszawa, Poland, tel: +48 2234340 50, fax: +48 2284495 10, e-mail: maciag_o@poczta.onet.pl 
( $>4$ ) and in patients who need system upgrade with regaining the venous access. The expanding TLE techniques are more and more often used for treating complications of long-lasting CIEDs therapy. The aim of the study was a retrospective analysis of the effectiveness and safety of TLE procedures concerning the leads implanted for at least 10 years.

\section{Methods}

Between 2008 and 2012 we performed TLE of 364 electrodes in 217 patients. Out of this $66(18.1 \%)$ leads in $43(19.8 \%)$ patients had been implanted for at least 10 years. The clinical data of patients were extracted from the subsequent TLE procedures database according to aforementioned criteria. The clinical characteristics of the study group are presented in Table 1.

The characteristics of the leads implanted at least 120 months before the TLE procedure were as follows: 58 pacing leads ( 9 active- and 49 passive-fixation), 8 defibrillating leads -7 endocardial (5 single-coil and 2 dual-coil leads; 2 active- and 5 passive-fixation) and 1 "floating" defibrillation lead placed in the superior vena cava.

The indications for TLE were: pocket infection in $24(56 \%)$ patients, lead excess and the necessity for regaining the venous access in 15 (38\%) patients and the lead-related infective endocarditis in the remaining $4(6 \%)$ patients.

The procedures were performed in operating or hybrid-operating room equipped with a highquality fluoroscopy, in general anesthesia and with cardiac surgery standby. In pacing-dependent patients the femoral temporary pacing was used.

All the adhesions and sutures in the device pocket were surgically prepared. The step-by-step approach was used from less advanced to more aggressive: manual direct traction, device traction with mechanical telescopic sheaths, autorotational cutting sheaths and femoral approach. The manual direct traction (simple traction) after stylet insertion was attempted for the leads, which were moving freely in the vessel. The most frequently employed technique was device traction - with the use of mechanical telescopic sheaths (stainless steel, PTFE - polytetrafluoroethylene and polypropylene) - Cook Vascular (Leechburg, PA, USA), which were rotated manually along the lead body to cut off the adhesions between the electrode and the vascular wall (Fig. 1). The locking stylet blocked in the intracardiac part of the lead was used to achieve its stiffness and to conduct a safe
Table 1. Clinical characteristics (number of patients $=43$ ).

\begin{tabular}{lc}
\hline Age [years] & $63(22-92)$ \\
Female & $14(32.6 \%)$ \\
NYHA class: & \\
I & $17(39.5 \%)$ \\
II & $20(46.5 \%)$ \\
III & $6(14 \%)$ \\
Primary disease/indication & $1(2.3 \%)$ \\
for implantation & \\
Long QT syndrome & \\
Arrhythmogenic RV cardiomyopathy & $1(2.3 \%)$ \\
Vasovagal syndrome & $1(2.3 \%)$ \\
Idiopathic ventricular fibrillation & $2(4.7 \%)$ \\
Hypertrophic cardiomyopathy & $2(4.7 \%)$ \\
Post-infarction HF & $4(9.3 \%)$ \\
HF with AV complete heart block & $5(11.6 \%)$ \\
Sick sinus syndrome & $11(25.6 \%)$ \\
AV heart block $-2^{\text {nd }}$ or $3^{\text {rd }}$ degree & $16(37.2 \%)$ \\
Type of previously implanted device: & \\
ICD with CRT & $1(2.3 \%)$ \\
Dual chamber ICD & $4(9.3 \%)$ \\
Single chamber ICD & $5(11.7 \%)$ \\
Single chamber pacemaker & $13(30.2 \%)$ \\
- atrial or ventricular & \\
Dual chamber pacemaker & $20(46.5 \%)$ \\
Number of previously performed & \\
procedures*: & $18(2.3 \%)$ \\
1 & $20(46.5 \%)$ \\
2 & $18(7 \%)$ \\
3 & $1(2.3 \%)$ \\
4 & \\
5 & \\
\hline
\end{tabular}

*Covers system implantations, exchanges and up-grade procedures; NYHA - New York Heart Association; RV - right ventricular; HF — heart failure; AV — atrioventricular; ICD — implantable cardioverter-defibrillator; CRT — cardiac resynchronization therapy

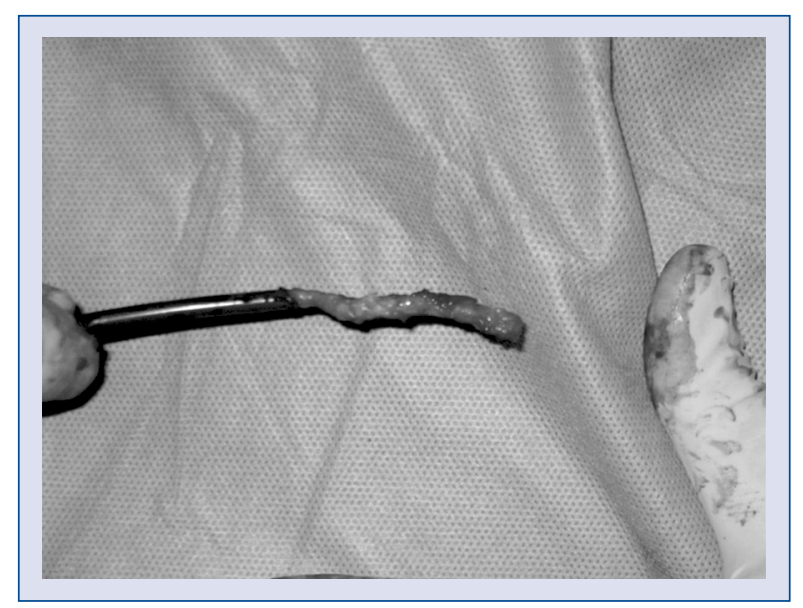

Figure 1. The tip-ending of the 26-year-old pacing lead extracted with the use of polytetrafluoroethylene telescopic sheaths; the surface of the lead is covered with the calcified connective tissue. 


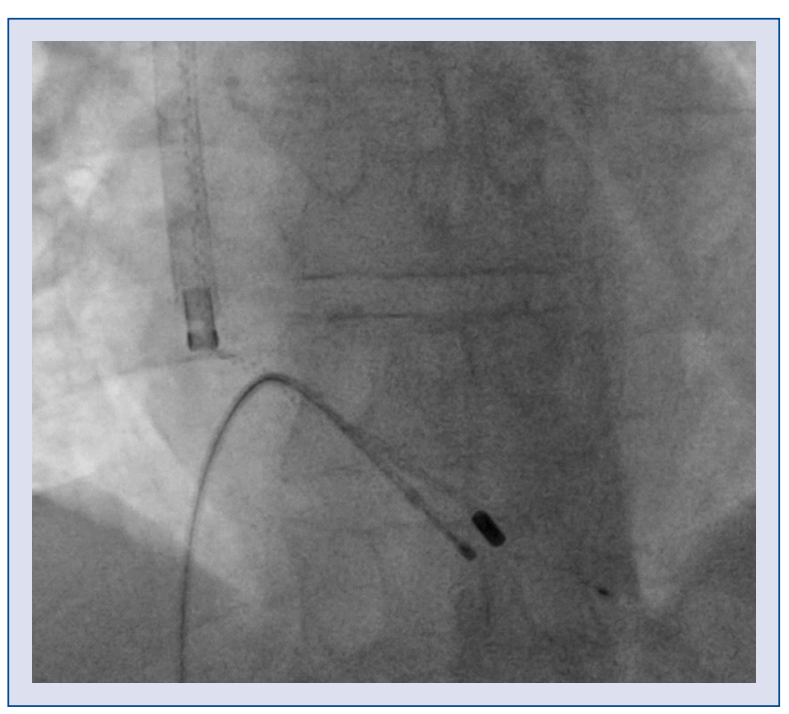

Figure 2. The example of using the "Evolution" autorotational system during the extraction of the pacing lead from the right ventricle.

preparation of the adhesions. The anchoring of the stylet was possible to be performed in the majority of the electrodes with the preserved inner lumen.

In 2 cases the autorotational cutting sheaths "Evolution" - Cook Vascular (Leechburg, PA, USA) (Fig. 2) were used to separate the adhesions, which could not be overcome with polypropylene sheaths — the used methodology was described previously [1]. In 1 case the femoral approach with the Needle's Eye Snare - Cook Vascular (Leechburg, PA, USA) was used to extract the lead, which was broken during the attempted subclavian TLE [2].

During the procedures performed on noninfected systems, after the lead extraction the guidewire was introduced to regain the venous access for the new implantation.

\section{Results}

The mean dwelling time was 161 months (median 144 months). The longest time for pacing and defibrillating lead was 330 and 251 months, respectively (Fig. 3).

The manual direct traction (simple traction) after stylet insertion was successful in $4(6 \%)$ leads. The most frequently applied technique was device traction - with the use of mechanical telescopic sheaths. Clinical success was achieved in next 59 (89\%) leads. In the remaining cases the autorotational cutting sheaths (2 leads; $3 \%$ ) and femoral approach (1 lead; 1.5\%) were successfully used.

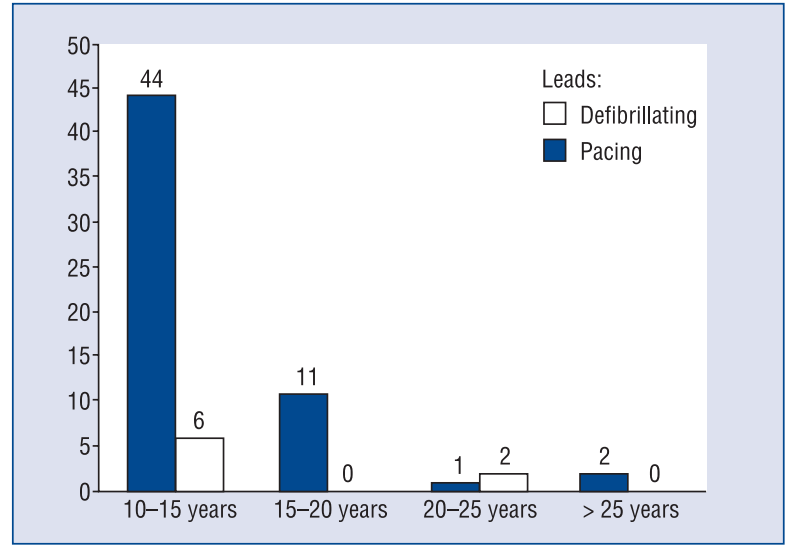

Figure 3. The number of extracted pacing and defibrillating leads in respective 5 -year intervals from the implantation.

Table 2. Results of the procedures.

\begin{tabular}{|c|c|}
\hline \multicolumn{2}{|l|}{ 1. Result $(I=66)$} \\
\hline Complete procedural success & $63 \mid(95 \%)$ \\
\hline Clinical success $(<2 \mathrm{~cm}$ of the lead left) & $3 \mid(5 \%)$ \\
\hline \multicolumn{2}{|l|}{ 2. Finally effective techniques $(I=66)$} \\
\hline Direct traction & $4 \mid(6 \%)$ \\
\hline Telescopic sheaths & $\begin{array}{c}591 \\
(89.5 \%)\end{array}$ \\
\hline Evolution mechanical system* & 2 I (3\%) \\
\hline Femoral access* & $1 \mathrm{I}(1.5 \%)$ \\
\hline \multicolumn{2}{|l|}{ 3. Complications ( $n=43$ ) } \\
\hline Major & 0 \\
\hline \multicolumn{2}{|l|}{ Minor: } \\
\hline Blood loss treated with transfusion & 2 pt $(4.7 \%)$ \\
\hline Peritoneal injury & $1 \mathrm{pt}(2.3 \%)$ \\
\hline
\end{tabular}

*Both methods used after attempt of telescopic sheaths extraction; I - leads; pt - patient; $\mathrm{n}$ - number of patients

Additionally, 10 leads implanted for less than 120 months were extracted during the procedures. The mean number of extracted leads in 1 patient was $1.8(1-4)$.

The intended clinical success was achieved in all patients. The complete procedural success rate was 95\% (63 leads) and in the remaining 3 (5\%) cases the small portion of the lead $(<2 \mathrm{~cm})$ with no clinical consequence was left in the vascular space. The results of the procedures are presented in Table 2.

Fluoroscopy time was available only for last 33 patients. Time was reached from 1 to $43 \mathrm{~min}$ (mean $16.6 \mathrm{~min}$ ). But there was no correlation between fluoroscopy time and dwelling time (Fig. 4). 


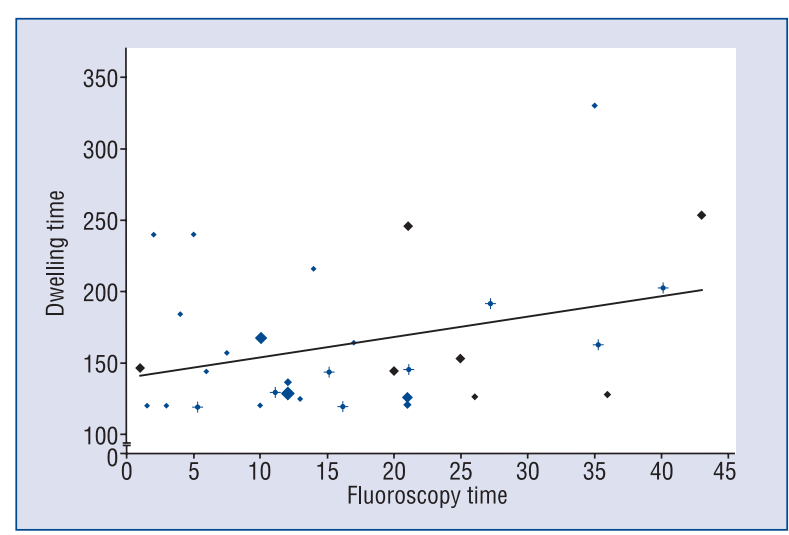

Figure 4. Comparison electrodes dwelling time with fluoroscopic time, amount of electrodes per patient figures by marker size ( 1 lead $\phi$, 2 to 4 leads $\diamond-\downarrow$ ), patients with implantable cardioverter-defibrillator leads marked with black color $(\bullet)$. Correlation without statistical significance.

No major procedural complications, such as death, vascular avulsion or tear, cardiac tamponade with rescue sternotomy, significant pulmonary embolism or stroke, were observed. In 2 cases intraprocedural blood loss was treated with transfusion. In 1 patient the peritoneal cavity was opened $(2-3 \mathrm{~cm})$ during the surgical preparation of infected tissues in the abdominal pocket (under the rectus abdominis muscle) of oldest implantable cardioverter-defibrillator system in our series. It was sutured with no further sequelae.

\section{Discussion}

The progress in medicine leads to the significant prolongation of patients' lives. It concerns CIEDs patients as well. Over the years they are referred to the scheduled device replacement and the upgrade of the system due to the still expanding indications. Although the patients experience treatment-related complications, the TLE techniques are more and more commonly used in dealing with the complications of CIEDs therapy [3-6].

CIED-related infection is an unequivocal class IB indication for the total system removal. As the non-infectious TLE procedures are class II indications, the decision should always depend on the number of existing leads, the expected patient's survival time and the prospective long-term complications [7-9]. The implantation of the next defibrillating lead, possibly interfering with the previously implanted one, may result in its significantly shorter functioning and inappropria- te interventions, especially in young individuals. It also increases the risk of vascular occlusion and may impede further procedures, which would require the central venous access [10].

Non-functional leads left in the vascular space increase the risk of infection as the insulation breakage facilitates bacterial colonization of the lead and inhibits the penetration of the antibiotic therapy [8, 11-13].

The extent of TLE procedure difficulty depends much on the time passed since the lead implantation. In large groups of patients, including also those with leads older than 10 years, the complication rate varies from $1 \%$ to $5 \%$. The most serious complications, resulting in the need for surgical intervention or death are reported in about $2 \%$ of patients. We did not observe major complications in the study group, but patients were completely prepared for thoracotomy and all the procedures were performed in general anesthesia, with the full stand-by of the cardiac surgeon [9, 14-18].

We believe that one of the key elements that resulted in the high efficacy of TLE in the study group was the possibility of using different extraction devices; and the crucial effect of lead stiffening with standard or locking stylets. Similarly to the other centers with vast experience in TLE, we most often used the mechanical telescopic sheaths, which were manually advanced over the lead and provided the high efficacy and safety of the procedures. Furthermore, it seems that such device enables gentler dosage of the force needed for cutting off the adhesions. It is not possible in the mechanical systems, where this force is enhanced by the gear system $[9,15,19]$.

The mechanical autorotating sheaths may be useful in the cases with massive adhesions or calcification, which are not always related to the oldest leads, but rather to the individual feature of the patient $[1,20,21]$.

The lack of the possibility of using laser or electrosurgical systems in our center did not disturb the achievement of the high procedural success rate with a low complication risk [22].

It is interesting that in the group of leads implanted more than 10 years before the extraction, some of them were able to be removed with a simple direct traction. Those leads were more likely to be defibrillating leads, due to their stiffness and resistance to extension and breaking. The percentage of such leads in the study group was small, whereas de Bie et al. [23] reported the (82\%) efficacy of direct traction in large group of patients with electrodes implanted for up to 84 months [19, 24]. 
The connective tissue, which binds the leads to the wall of the vessel, heart or the valve apparatus, is not formed evenly in all patients. The quantity of this tissue depends on the thrombus formation on the lead, as well as the individual collagen type and calcium metabolism causing the calicification of the connective tissue bands. This mechanism was described on the basis of histological findings and autopsies of patients with CIEDs [25, 26].

In this study group the femoral access was performed in the case, where subclavian TLE was unsuccessful. Such approach is used in some centers, while the others prefer the femoral access as a first choice method of TLE. This way of lead extraction seems to be faster and of comparable safety. One should remember however, that the growing population of non-infectious patients suggests performing TLE in the primary implantation site to regain the venous access [27, 28].

\section{Limitations of the study}

A low number of patients may be not enough to identify seldom complications like superior vena cava tear or tamponade. Lack of procedure and fluoroscopy time did not allow fully desired statistics.

\section{Conclusions}

Transvenous extraction of the leads which have been implanted in the vascular space for over 10 years is an effective and safe method of treating infectious, vascular and mechanical complications of CIEDs therapy.

Conflict of interest: A. Maciąg, P. Sysyka, A. Przybylski, M. Sterliński, A. Oręziak — travelling grant HammerMed/Cook Medical; A. Przybylski — proctoring contract HammerMed/Cook Medical; K. Kuśmierski, M. Pytkowski, J. Różański, H. Szwed - no conflict.

\section{References}

1. Przybylski A, Syska P, Oręziak A et al. Usefulness of the evolution mechanical dilator sheath for endocardial lead extraction: Preliminary results. Post Kardiol Interw, 2010; 6: 59-65.

2. Kuśmierski K, Syska P, Maciąg A, Oręziak A, Kuśmierczyk M, Przybylski A. Regaining venous access for implantation of a new lead. Post Kardiol Interw, 2013; 9: 16-21.

3. Kutarski A, Pietura R, Młynarczyk K, Małecka B, Głowniak A. Pacemaker lead extraction and recapture of venous access: Technical problems arising from extensive venous obstruction. Cardiol J, 2012; 19: 513-517.

4. Rusanov A, Spotnitz HM. A 15-year experience with permanent pacemaker and defibrillator lead and patch extractions. Ann Thorac Surg, 2010; 89: 44-50.
5. Kennergren C, Bjurman C, Wiklund R, Gäbel J. A single-centre experience of over one thousand lead extractions. Europace, 2009; 11: 612-617.

6. Jones SO 4th, Eckart RE, Albert CM, Epstein LM. Large, single-center, single-operator experience with transvenous lead extraction: outcomes and changing indications. Heart Rhythm, 2008; 5: 520-525.

7. Wilkoff BL, Love CJ, Byrd CL et al. Heart Rhythm Society; American Heart Association. Transvenous lead extraction: Heart Rhythm Society expert consensus on facilities, training, indications, and patient management: This document was endorsed by the American Heart Association (AHA). Heart Rhythm, 2009; 6: 1085-1104.

8. Bongiorni MG, Marinskis G, Lip GY, Svendsen JH, Dobreanu D, Blomström-Lundqvist C; Scientific Initiative Committee, European Heart Rhythm Association. How European centres diagnose, treat, and prevent CIED infections: Results of an European Heart Rhythm Association survey. Europace, 2012; 14: 1666-1669.

9. Bongiorni MG, Blomström-Lundqvist C, Kennergren C et al. Scientific Initiative Committee, European Heart Rhythm Association. Current practice in transvenous lead extraction: A European Heart Rhythm Association EP Network Survey. Europace, 2012; 14: 783-786.

10. Bode F, Himmel F, Reppel M, Mortensen K, Schunkert H, Wiegand UK. Should all dysfunctional high-voltage leads be extracted? Results of a single-centre long-term registry. Europace, 2012; 14: 1764-1770.

11. Kolodzinska K, Kutarski A, Grabowski M, Jarzyna I, Małecka B, Opolski G. Abrasions of the outer silicone insulation of endocardial leads in their intracardiac part: A new mechanism of lead-dependent endocarditis. Europace, 2012; 14: 903-910.

12. Kutarski A, Trojnar M, Tomaszewski A, Oleszczak K, Głowniak A. Diagnosis and treatment of lead-dependent infective endocarditis. Kardiol Pol, 2011; 69: 502-504.

13. Silvetti M, Drago F. Outcome of young patients with abandoned, nonfunctional endocardial leads. PACE, 2008; 31: 473-479.

14. Arujuna A, Williams S, Whittaker J et al. Trends, indications and outcomes of cardiac implantable device system extraction: A single UK centre experience over the last decade. Int J Clin Pract, 2012; 66: 218-225.

15. Kutarski A, Małecka B, Ruciński P, Zabek A. Percutaneous extraction of endocardial leads: A single centre experience in 120 patients. Kardiol Pol, 2009; 67: 149-156.

16. Deharo JC, Bongiorni MG, Rozkovec A et al. European Heart Rhythm Association. Pathways for training and accreditation for transvenous lead extraction: A European Heart Rhythm Association position paper. Europace, 2012; 14: 124-134.

17. Williams SE, Arujuna A, Whitaker J et al. Percutaneous extraction of cardiac implantable electronic devices (CIEDs) in octogenarians. Pacing Clin Electrophysiol, 2012; 35: 841-849.

18. HauserRG,Katsiyiannis WT, GornickCC,AlmquistAK, KallinenLM. Deaths and cardiovascular injuries due to device-assisted implantable cardioverter-defibrillator and pacemaker lead extraction. Europace, 2010; 12: 395-401.

19. Di Cori A, Bongiorni MG, Zucchelli G et al. Transvenous extraction performance of expanded polytetrafluoroethylene covered ICD leads in comparison to traditional ICD leads in humans. Pacing Clin Electrophysiol, 2010; 33: 1376-1381.

20. Maytin M, Epstein LM, John RM. Lead implant duration does not always predict ease of extraction: Extraction sheath may be required at $<1$ year. Pacing Clin Electrophysiol, 2011; 34: 1615-1620. 
21. Oto A, Aytemir K, Canpolat U et al. Evolution in transvenous extraction of pacemaker and implantable cardioverter defibrillator leads using a mechanical dilator sheath. Pacing Clin Electrophysiol, 2012; 35: 834-840.

22. Kennergren C, Bucknall CA, Butter C et al. Laser-assisted lead extraction: The European experience. Europace, 2007; 9: 651-656.

23. de Bie MK, Fouad DA, Borleffs CJ et al. Trans-venous lead removal without the use of extraction sheaths, results of $>250$ removal procedures. Europace, 2012; 14: 112-116.

24. Małecka B, Kutarski A, Grabowski M. Is the transvenous extraction of cardioverter-defibrillator leads more hazardous than that of pacemaker leads? Kardiol Pol, 2010; 68: 884-890.

25. Kołodzińska A, Kutarski A, Koperski Ł, Grabowski M, Małecka B, Opolski G. Differences in encapsulating lead tissue in patients who underwent transvenous lead removal. Europace, 2012; 14: 994-1001.

26. Dvorak P, Novak M, Kamaryt P, Slana B, Lipoldova J, Dvorak P. Histological findings around electrodes in pacemaker and implantable cardioverter-defibrillator patients: Comparison of steroid-eluting and non-steroid-eluting electrodes. Europace, 2012; 14: 117-123.

27. Bracke FA, Dekker L, van Gelder BM. The Needle's Eye Snare as a primary tool for pacing lead extraction. Europace, 2013; 15: 1007-1012.

28. Bordachar P, Defaye P, Peyrouse E et al. Extraction of old pacemaker or cardioverter-defibrillator leads by laser sheath versus femoral approach. Circ Arrhythm Electrophysiol, 2010; 3: 319-323. 